\title{
Combinatorial Enzyme Approach to Produce Oligosaccharides of Diverse Structures for Functional Screen
}

\author{
Dominic W. S. Wong*, Doris Feng, Sarah Batt, William Orts \\ Western Regional Research Center, USDA-ARS, Albany, CA, USA \\ Email: *Dominic.Wong@ars.usda.gov
}

How to cite this paper: Wong, D.W.S., Feng, D., Batt, S. and Orts, W. (2018) Combinatorial Enzyme Approach to Produce Oligosaccharides of Diverse Structures for Functional Screen. Advances in Enzyme Research, 6, 11-20.

https://doi.org/10.4236/aer.2018.62002

Received: June 21, 2018

Accepted: June 26, 2018

Published: June 29, 2018

Copyright $\odot 2018$ by authors and Scientific Research Publishing Inc. This work is licensed under the Creative Commons Attribution International License (CC BY 4.0).

http://creativecommons.org/licenses/by/4.0/

\begin{abstract}
Combinatorial chemistry has been a focus of research activity in modern drug discovery and biotechnology. It is a concept by which a vast library of molecular diversity is synthesized and screened for target properties. This report is to illustrate the application of enzyme technology using the concept of combinatorial chemistry as a novel approach for the bioconversion of plant fibers. Citrus pectin was subjected to combinatorial enzyme digestion to create libraries of pectic oligosaccharides with diverse structural variants. Repeated cycles of fractionation and screening resulted in the isolation and identification of an active oligoGalA species with antimicrobial activity.
\end{abstract}

\section{Keywords}

Combinatorial Enzyme, Polygalacturonase, Pectate Lyase, Pectin,

Oligosaccharides

\section{Introduction}

The central idea of combinatorial chemistry is to synthesize a diverse population of the molecule of interest and screen the population for the few variants that exhibit the desirable and useful property. A simple organic molecule with a core structure carrying five randomly arranged substitutions theoretically leads to a total of 3125 structural variants. A pentapeptide library randomized with the 20 natural amino acids at each position will produce a combinatorial library of 3.2 million distinct peptides. The technology of combinatorial chemistry has been extensively reviewed for its applications in drug discovery and biotechnology [1] [2], in agrosciences [3], and in agriculture and food [4] [5]. The key techniques for creating combinatorial libraries can be divided into chemical and biology 
approaches. The former includes solid-phase synthesis and its refinement for libraries of small organic molecules, peptides, and oligonucleotides. The biological approach uses the genetic code as a precursor to express randomized libraries in microorganisms, such as phage, E. coli, and yeast. A recent development in this aspect is the creation of dynamic combinatorial chemistry by which the library is evolved under thermodynamic control [6].

Plant cell wall polysaccharides consist of polymeric backbones decorated with various types of substitutions. For example, xylan contains a $\beta$-1,4-linked xylopyranosyl chain decorated with at least six types of side groups. Likewise, xyloglucans, pectins and others consist of varieties of side group substitutions that can be targeted by specific enzymes. The presence of these side groups as well as their positions, density, and types of linkages influences the pattern of enzymatic degradation of the main chain polymer and vice versa. These cooperative interactions determine the structural outcome of the oligosaccharide fragments produced. The use of enzymes to surgically remove the side group moieties individually and/or sequentially, therefore, constitute a combinatorial design for generating vast libraries of structurally diverse oligosaccharides that would translate into different and unique reactivity and functional properties. The diverse population can often be screened with high-throughput methods for candidates possessing the target biological and/or functional properties. This combinatorial enzyme approach should be applicable for exploration of new structures and functions in all aspects of biopolymer degradation. To confirm the novel concept of "combinatorial enzyme technology", we applied the design to produce libraries of pectic oligosaccharides from citrus pectin. Repeated fractionation and screening resulted in the isolation of an active pectic oligosaccharide species with antimicrobial activity (Figure 1).

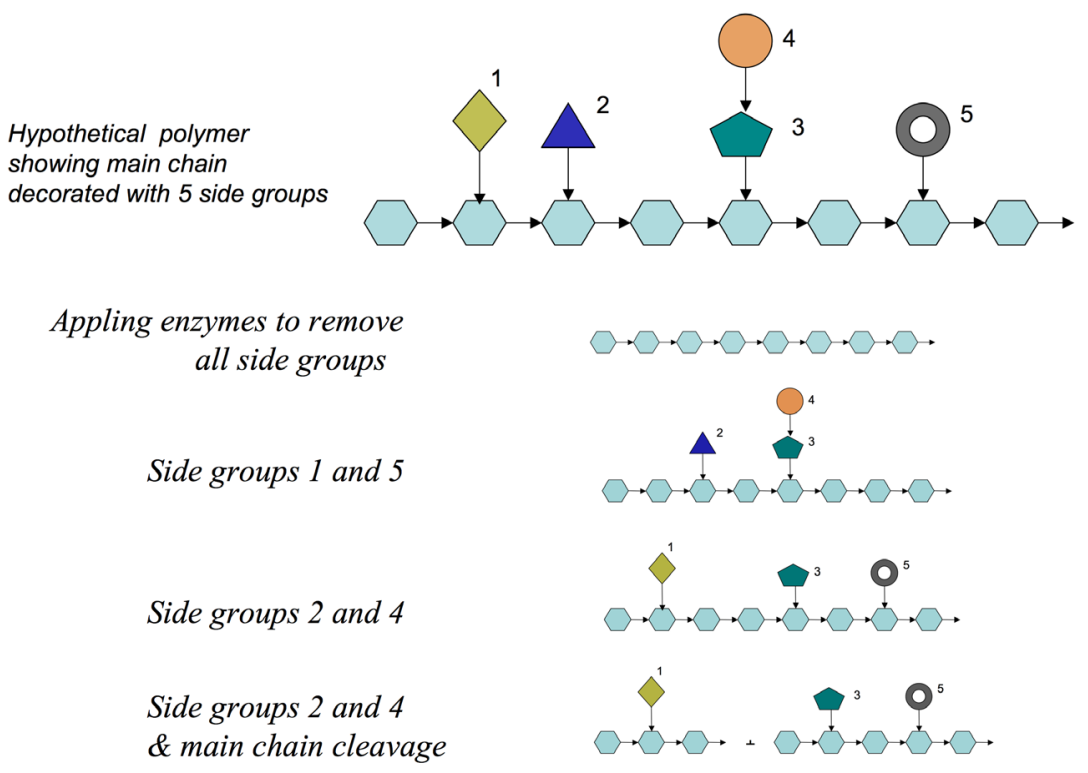

Figure 1. A simplified schematic representation of combinatorial enzyme digestion to produce libraries of oligosaccharides of diverse structures. 


\section{Materials and Methods}

\subsection{Materials}

Citrus pectin was obtained from Sigma (St. Louis, MO), washed 3x with ethanol and dried in a $50^{\circ} \mathrm{C}$ oven. Galacturonic acid, diGalA, and triGalA were purchased from Sigma (St. Louis, MO). All pectinolytic enzymes were obtained from Megazyme (Wicklow, Ireland). E. coli (ATCC 8739) was obtained from American Type Culture Collection (Manassas, VA). Culture media were purchased from Difco (Leeuwarden, The Netherlands). DEAE Sepharose FF resin and Sephadex G-10 column were obtained from GE Health Science (Pittsburg, PA). HPTLC plates were purchased from Analtech (Newark, DE). All chemicals and solvents were of analytical or HPLC grade.

\subsection{Enzymatic Hydrolysis of Pectin}

Citrus pectin was dissolved in water at a concentration of $1 \%$, adjusted with $\mathrm{NaOH}$ to $\mathrm{pH}$ 5.0. Polygalacturonase (300 U) was added, incubated at $30^{\circ} \mathrm{C}$ at two time points. The reaction mixtures were cooled to room temperature, adjusted to $\mathrm{pH} 8.0$, combined and mixed thoroughly. Calcium chloride was added to a final concentration of $2 \mathrm{mM}$, and the mixture was separated into equal volumes to which four dosages of pectate lyase (50 to $400 \mathrm{U}$ ) were added and incubated in a shaking water bath. At each time interval, the reaction mixture was adjusted to $\mathrm{pH} 2.0$. Ethanol was added at 1:1 v/v ratio followed by centrifugation to remove the precipitates. The supernatant collected was heated to $80^{\circ} \mathrm{C}$ for 10 min to inactivate the enzymes. The ethanol was removed by vacuum evaporation and then lyophilized.

\subsection{High-Performance Thin-Layer Chromatography}

The pectic oligo sample was applied to $20 \times 10 \mathrm{~cm}$ HPTLC silica gel F254 plate and developed with EtOAc/HOAc/1-PrOH/HCOOH/ $\mathrm{H}_{2} \mathrm{O}$ (25:10:5:1:15). The unsaturation of oligo fragments was observed by spraying with $\mathrm{KMnO}_{4}$ reagent ( $3 \mathrm{~g} \mathrm{KMnO}_{4}, 20 \mathrm{~g} \mathrm{~K}_{2} \mathrm{CO}_{3}, 5 \mathrm{ml} 5 \%$ aqueous $\mathrm{NaOH}$, and $300 \mathrm{ml} \mathrm{H}_{2} \mathrm{O}$ ). The plate was then sprayed with $10 \% \mathrm{H}_{2} \mathrm{SO}_{4}$ in methanol containing $1 \mathrm{mg} / \mathrm{ml}$ orcinol, followed by heating at $90^{\circ} \mathrm{C}$ for visualization of oligosaccharides.

\subsection{Ion-Exchange Chromatography}

Anion separation was performed at room temperature using a DEAE Sepharose FF column $(2.5 \times 60 \mathrm{~cm})$. The enzyme digest $(2 \mathrm{~g}$ in $50 \mathrm{ml}$ water $)$ was first adjusted to $\mathrm{pH} 5.0$ and then applied onto the column, followed by elution with a gradient from 0 to $0.7 \mathrm{M} \mathrm{NaCl}$. The flow rate was $0.5 \mathrm{ml} / \mathrm{min}$. The fractions were measured for unsaturation by $\mathrm{A}_{235}$ reading, for reducing sugars by the DNSA method [7], and for total carbohydrates by the phenol-sulfuric acid method [8].

\subsection{HPLC Analysis}

Analysis of the active oligoGalA species was performed on a Shimadzu LC-10 
AD system fitted with a UV-vis detector, using an anion-exchange Sorbax SAX column $(4.6 \times 250 \mathrm{~mm})$ with $0.1 \mathrm{M}$ sodium acetate $\mathrm{pH} 5.4$ as the mobile phase at a flow rate of $0.3 \mathrm{ml} / \mathrm{min}$ at room temperature. Gel permeation chromatography (GPC) was performed using an Ultrahydrogel column $(7.8 \times 300 \mathrm{~mm})$, with water as the mobile phase at a flow rate of $0.3 \mathrm{ml} / \mathrm{min}$ run. Pullulan standards were used for MW calibration.

\subsection{Growth Experiments and Culture Conditions}

A freeze-dried E. coli (ATCC 8739) was rehydrated with $1 \mathrm{ml}$ sterile Difco ${ }^{\mathrm{TM}} \mathrm{MH}$ (Mueller Hinton) broth and streaked onto a $\mathrm{MH}$ agar plate. A $5 \mathrm{ml}$ overnight culture was prepared and the absorbance at $600 \mathrm{~nm}$ was measured. The culture was diluted with $\mathrm{MH}$ broth to a final concentration of $1 \times 10^{3} \mathrm{cfu} / \mathrm{ml}$ based on a standard curve. The active pectic oligoGalA species was dissolved in $\mathrm{MH}$ broth at specific concentrations, filter sterilized $(2 \mu \mathrm{m}$ HT Tuffryn membrane syringe filter, Pall Corporation), and added to the diluted $E$. coli culture. Cell growth was determined by measuring the absorbance at $600 \mathrm{~nm}$ of appropriate dilutions of the cell culture using microplate reader (SpectraMax M2, Molecular Devices, $\mathrm{CA}$ ). The oligo concentration was determined by the phenol-sulfuric acid method based on standard curve of galacturonic acid. Minimum inhibitory concentration (MIC) was determined by the standard microdilution method. It is defined as the lowest concentration of an antimicrobial that inhibits the visible growth of a microorganism after overnight incubation [9] [10].

\section{Results and Discussion}

The reaction mixture obtained after combinatorial enzyme digestion was fractionated by ethanol precipitation to yield the pectic oligosaccharides separated from the higher molecular weight polymeric pectins. The yield was about $40-60$ percent. The oligosaccharides showed varying degrees of chain length and unsaturation on HPTLC separation (Figure 2).

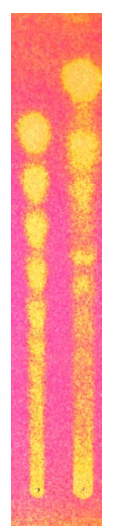

Figure 2. HPTLC separation of enzyme digest of citrus pectin. Lane 1: corn syrup; Lane 2: pectic oligosaccharides. Samples were spotted on silica GHL plate, developed in ethyl acetate/acetic acid/1-propanol/ $\mathrm{HCOOH} / \mathrm{H}_{2} \mathrm{O}$ (25:10:5:1:15), and sprayed with $\mathrm{KMnO}_{4}$ reagent as described in "Methods". 
The oligosaccharides were applied to a DEAE Sepharose FF column, which separated the pectic digest into major fractions: A, B, C and D, which were all unsaturated (showing absorbance at $235 \mathrm{~nm}$ ) and showed positive with the phenol- $\mathrm{H}_{2} \mathrm{SO}_{4}$ assay (suggesting carbohydrate detection) (Figure 3). However, in contrast to the others fraction $\mathrm{D}$ (pooled fractions 92 - 105) did not react with DNSA suggesting it was not a reducing sugar. From the elution pattern in the chromatogram, the pectic oligo fraction $\mathrm{D}$ was relatively more anionic than the other three fractions.

The pectic oligo fraction $\mathrm{D}$ showed an unexpected inhibitory effect on the growth of the E. coli strain ATCC 8739. This active oligoGalA species was recovered and lyophilized, and further analyzed by HPLC using anion exchange and gel permeation columns. It consisted of essentially one major peak on the HPLC gel permeation column using RI and UV235 detectors (Figure 4).

The screening of the enzyme-derived oligosaccharides led to the detection of antimicrobial activity in an active oligoGalA species. In each set of experiments, the initial inoculation of the microorganism was carefully controlled to $0.5 \times 10^{3}$ $\mathrm{cfu} / \mathrm{ml}$ titer, so that the comparisons were performed under same starting conditions. Figure 5 shows that the inhibitory effect increased with the concentration, and a complete suppression of cell growth was achieved at $0.4 \% \mathrm{w} / \mathrm{v}$, which was the MIC (minimum inhibitory concentration) value. The antimicrobial effect was sustainable for at least three days and possibly longer (Figure 6).

The mechanism of inhibition might be related to the unique structural properties of the active species. It had a highly unsaturated structure due to the elimination reaction of pectate lyase, as confirmed by the absorbance measurement at $235 \mathrm{~nm}$. The active oligo species was also highly sensitive to permanganate oxidation, yielding a bright yellow band with a pink color background on

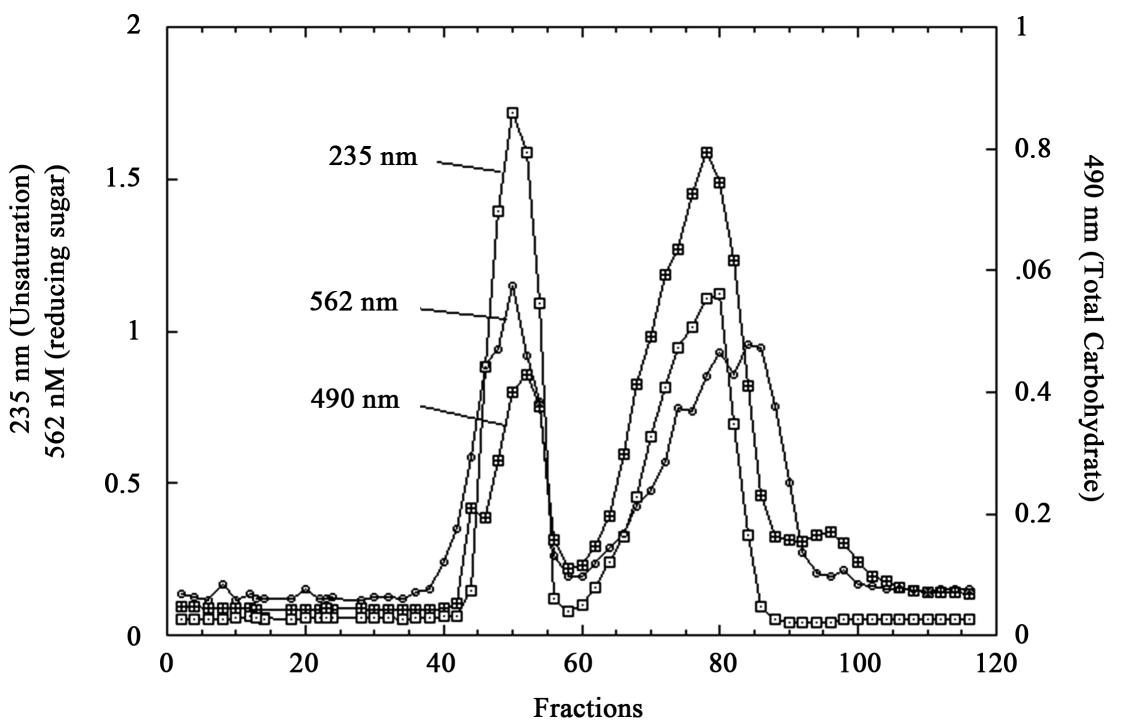

Figure 3. Anion-exchange chromatographic fractionation of enzyme-digested citrus pectin. Column: DEAE Sepharose FF in $2.5 \times 60 \mathrm{~cm}$, Flow rate: $0.5 \mathrm{ml} / \mathrm{min}$, Gradient elution: 0 to $0.7 \mathrm{M} \mathrm{NaCl}$. 


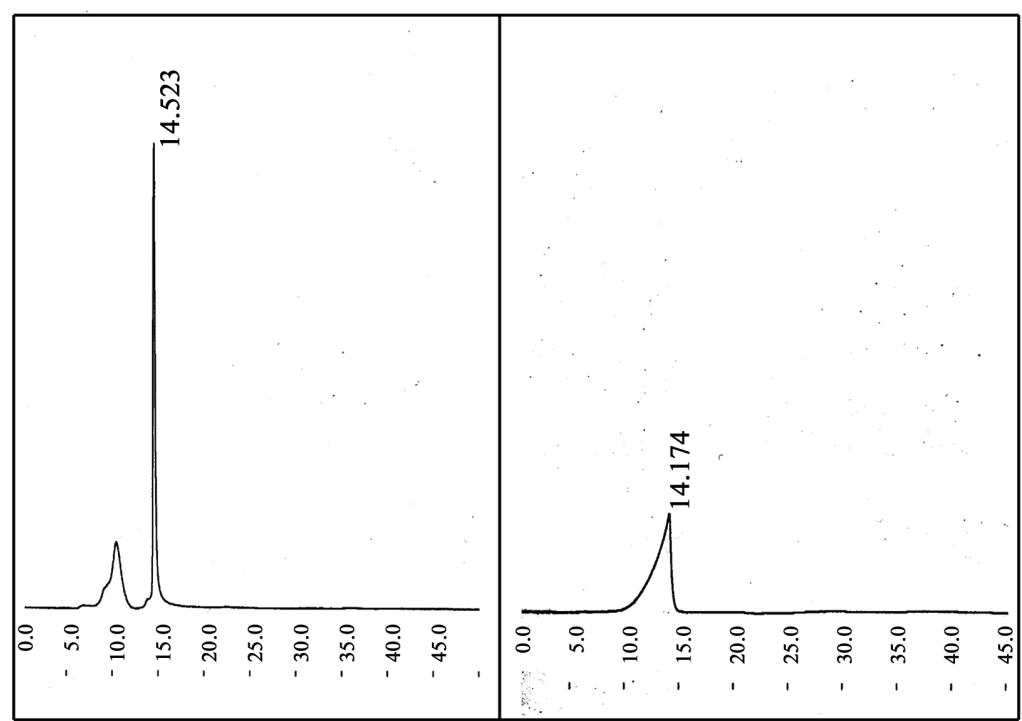

(a)

(b)

Figure 4. HPLC analysis of the active oligoGalA species. Analysis was performed on a Shimadzu LC-10 AD system fitted with a uv-vis detector and a RI detector, using (a) Sorbax SAX column $(4.6 \times 250 \mathrm{~mm})$. The mobile phase was $0.1 \mathrm{M}$ sodium acetate $\mathrm{pH} 5.4$ run at a flow rate of $0.3 \mathrm{ml} / \mathrm{min}$ at room temperature; (b) Ultrahydrogel gel 120 column, with $\mathrm{H}_{2} \mathrm{O}$ as mobile phase.

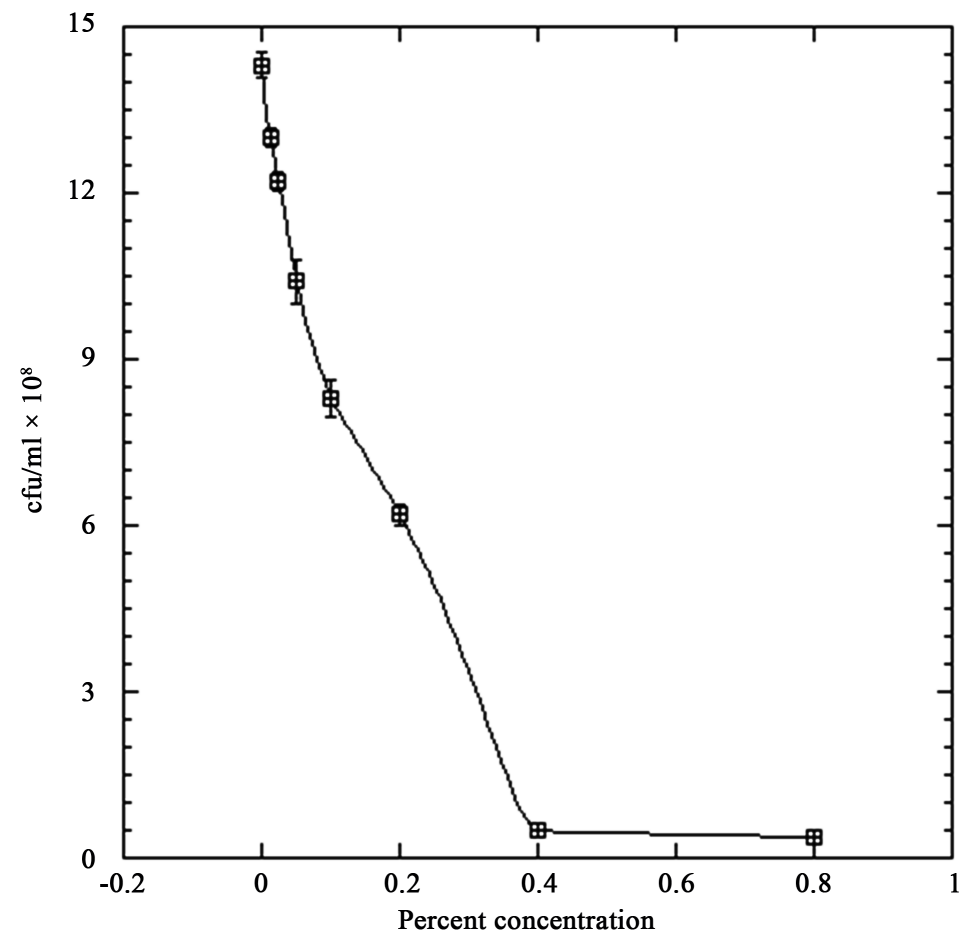

Figure 5. Increasing concentration effect of the active oligoGalA species on cell growth. An overnight culture of E. coli (ATCC 8739) in $\mathrm{MH}$ broth at $35^{\circ} \mathrm{C}$ was diluted to appropriate titer concentration, and various concentration of the oligoGalA $(0.005 \%$ to $0.8 \%)$ were added. The incubation was continued for $24 \mathrm{hr}$. Cell growth was determined by measuring $\mathrm{A}_{600}$ of appropriate dilutions of cell culture, and the cfu was calculated based on the conversion curve. 


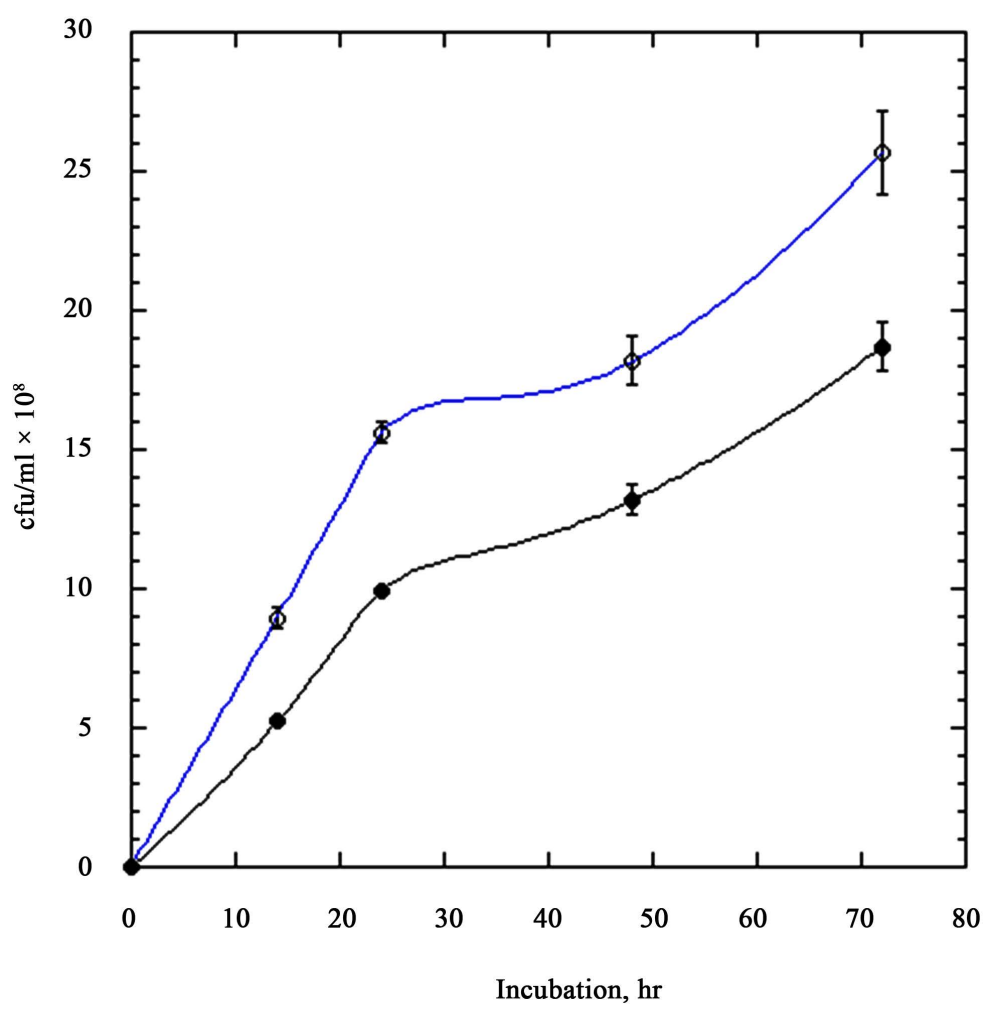

Figure 6. Time course of the effect of the active oligoGalA species on cell growth. An overnight culture of E. coli (ATCC 8739) in $\mathrm{MH}$ broth at $37^{\circ} \mathrm{C}$ was diluted to appropriate titer concentration, and the oligo species at $0.01 \%$ was added. The incubation was continued for 3 days with sampling every 12 and $24 \mathrm{hr}$. Cell growth was determined by measuring $\mathrm{A}_{600}$ of appropriate dilutions of the cell culture, and the $\mathrm{cfu} / \mathrm{ml}$ cell density was calculated based on the conversion curve.

HPTLC. Gel permeation chromatography suggests that the species had a size range of $1.2 \mathrm{kDa}$, generally considered in the range of low molecular weight oligosaccharides. The pectic hydrolysate consists mainly of acidic oligosaccharides because the parent structure of pectin has carboxylate side groups.

The presence of reactive double bonds in the active oligo species may be a contributing factor to its inhibitory effect on cell growth. The antimicrobial activity of some classes of phenolic compounds has been attributed to the presence of reactive double bonds, the association of the acid moiety, and the ability to facilitate membrane permeability [11]. Double bonds are electrophilic and readily participate in a variety of reactions, resulting in crosslinking and inactivation of biomolecules. Antimicrobial activities of phenolic compounds are found in hydrolysis of lignocellulosic materials, comparable to the common preservative sodium benzoate [12].

The small size of the oligosaccharide may be an important factor in facilitating passage through the cell membrane. Oligosaccharides in the high molecular weight range have been shown to prevent efficient in vivo utilization and not to exert functional and biological activities. Many antimicrobial oligosaccharides reported in the literature are low molecular weight molecules, consisting of tri-, 
tetra-, and pentamers [13] [14]. The present results seem to support the suggestion that small molecular size allows the penetration of cell membrane, and interactions with intracellular constituents and processes.

There have been reports that acidic (glucuronic acid-containing) xylans inhibit gram-positive bacteria [13]. Alginate oligosaccharides, consisting of guluronic and mannuronic acids in the main chain, have also been suggested to cause disruption of the formation of bacterial biofilms [15] [16]. Further investigation will be needed to relate the functional aspect of acidic groups in contributing to the antimicrobial effect.

The active pectic oligo species in the present study can be considered comparable to some of the common food preservatives, which are generally applied in the range of $0.1 \%$. The use of non-digestible oligosaccharides (NDO) has gained popularity as functional food ingredients. Oligosaccharides have also been promoted in recent years as alternatives for antibiotics as antimicrobial growth performance promoters (AGP) in animal production [17]. The health cause-effect of AGP and NDO is generally linked to the modification of the microflora, and thus the physiological conditions of the intestinal system [18]. In both regards, oligosaccharides are commonly used in sub-minimum inhibitor concentrations acting to modulate the microbiota composition.

\section{Conclusion}

A novel combinatorial enzyme approach has been developed to create libraries of oligosaccharides of diverse structures for functional screen. A species of enzyme-derived pectic oligosaccharide has been isolated and shown to suppress the growth of the test organism ATCC 8739. The MIC value was estimated to be $0.4 \%$, comparing favorably to other reported oligosaccharides and some common food preservatives. The active pectic oligo species may be useful as alternatives for antimicrobial growth promoters or a new source of high-value preservatives. The present study thus demonstrates the science and practical use of the combinatorial enzyme approach.

\section{Acknowledgements}

Reference to a company and/or products is only for purposes of information and does not imply approval of recommendation of the product to the exclusion of others that may also be suitable. All programs and services of the U.S. Department of Agriculture are offered on a nondiscriminatory basis without regard to race, color, national origin, religion, sex, age, marital status, or handicap. The authors declare that there is no conflict of interest regarding the publication of this paper.

\section{Conflicts of Interest}

The authors declare no conflicts of interest regarding the publication of this paper. 


\section{References}

[1] Kennedy, J.P., Williams, L., Bridges, T.M., Daniels, R.N., Weaver, D. and Lindsley, G.W. (2008) Application of Combinatorial Chemistry Science on Modern Drug Discovery. Journal of Combinatorial Chemistry, 10, 345-354. https://doi.org/10.1021/cc700187t

[2] Seneca, P., Fassina, G., Frecer, V. and Miertus, S. (2014) The Effects of Combinatorial Chemistry and Technologies on Drug Discovery and Biotechnology-A Mini Review. Nova Biotechnologica et Chimica, 13, 87-108. https://doi.org/10.1515/nbec-2015-0001

[3] Lindell, S.D., Pattenden, L.C. and Shannon, J. (2009) Combinatorial Chemistry in the Agrosciences. Bioorganic \& Medicinal Chemistry, 17, 4035-4046. https://doi.org/10.1016/j.bmc.2009.03.027

[4] Wong, D.W.S. and Robertson, G. (2004) Applying Combinatorial Chemistry and Biology to Food Research. Journal of Agricultural and Food Chemistry, 52, 7187-7198. https://doi.org/10.1021/jf040140i

[5] Wong, D.W.S. and Robertson, G. (1999) Combinatorial Chemistry and Its Applications in Agriculture and Food. In: Shahidi et al., Eds., Chemicals via Higher Plant Bioengineering, Kluwer Academic/Plenum Publishers, New York, 91-105. https://doi.org/10.1007/978-1-4615-4729-7_8

[6] Herrmann, A. (2014) Dynamic Combinatorial/Covalent Chemistry: A Tool to Read, Generate and Modulate the Bioactivity of Compounds and Compound Mixtures. Chemical Society Reviews, 43, 1899-1933. https://doi.org/10.1039/C3CS60336A

[7] Miller, G.L. (1959) Use of Dinitrosalicyclic Acid Reagent for Determination of Reducing Sugar. Analytical Chemistry, 31, 426-428.

https://doi.org/10.1021/ac60147a030

[8] Masuko, T., Minami, A., Iwasaki, N., Majima, T., Nishimura, S.-I. and Lee, Y.C. (2005) Carbohydrate Analysis by a Phenol-Sulfuric Acid Method in Microplate Format. Analytical Biochemistry, 239, 69-72.

https://doi.org/10.1016/j.ab.2004.12.001

[9] Andrews, J.M. (2001) Determination of Minimum Inhibitory Concentrations. Journal of Antimicrobial Chemotherapy, 48, 5-16. https://doi.org/10.1093/jac/48.suppl_1.5

[10] Nychas, G.J.E. (1995) Natural Antimicrobials from Plants. In: Gould, G.W., Ed., New Methods of Foods Preservation, Blackie Academic Chapman \& Hall, Glasgow, 53-83, 3-2, 87-108. https://doi.org/10.1007/978-1-4615-2105-1_4

[11] Garrote, G., Cruz, J.M., Moure, A., Dominguez, H. and Parajo, J.C. (2004) Antioxidant Activity of Byproducts from the Hydrolytic Processing of Selected Lignocellulosic Materials. Trends in Food Science \& Technology, 15, 191-200. https://doi.org/10.1016/j.tifs.2003.09.016

[12] Aziz, N.H., Farag, S.E., Mousa, L.A. and Abo-Zaid, M.A. (1998) Comparative Antibacterial and Antifungal Effects of Some Phenolic Compounds. Microbios, 93, 43-54.

[13] Christakopoulos, P., Katapodis, P., Kalogeris, E., Kekos, D., Macris, B.J., Stamatis, H. and Shaltsa, H. (2003) Antimicrobial Activity of Acidic Xylo-Oligosaccharides Produced by Family 10 and 11 Endoxylanases. International Journal of Biological Macromolecules, 31, 171-175. https://doi.org/10.1016/S0141-8130(02)00079-X

[14] Chaan, F., Belghith-Ferrdri, L., Zaouri-Ellouzi, S., Driss, D., Bilbech, M., Kallel, F., Bouaziz, F., Mehdi, Y., Ellouz-Chaabouni, S. and Ghorbel, R. (2016) Antibacterial and Antioxidant Properties of Mixed Linkage Beta-Oligosaccharides from Extracted 
$\beta$-Glucan Hydrolyzed by Penicillium occitanis $\mathrm{EG}_{\mathrm{L}}$ Lichenase. Natural Product Research, 30, 1353-1359. https://doi.org/10.1080/14786419.2015.1056185

[15] Khan, S., Tendervik, A., Sletta, H., Klinkenberg, G., Emanuel, C., Onseyen, E., Myrvold, R., Howe, R.A., Walsh, T.R., Hill, K.E. and Thomas, D.W. (2012) Overcoming Drug Resistance with Alginate Oligosaccharides Able to Potentiate the Action of Selected Antibiotics. Antimicrobial Agents Chemotherapy, 56, 5134-5141. https://doi.org/10.1128/AAC.00525-12

[16] Tendervik, A., Sletta, H., Klinkenberg, G., Emanuel, C., Powell, L.C., Pritchard, M.F., Khan, S., Craine, K.M., Onseyen, E., Rye, P.D., Wright, C., Thomas, D.W. and Hill, K.E. (2014) Alginate Oligosaccharides Inhibit Fungal Cell Growth and Potentiate the Activity of Antifungals against Candida and Aspergillus spp. PLOS One, 9, e112518. https://doi.org/10.1371/journal.pone.0112518

[17] Huyghebaert, G., Ducatelle, R. and Van Immerseel, F. (2011) An Update on Alternatives to Antimicrobial Growth Promoters for Broilers. The Veterinary Journal, 187, 182-188. https://doi.org/10.1016/j.tvjl.2010.03.003

[18] Mussatto, S.I. and Mancilha, I.M. (2007) Non-Digestible Oligosaccharides: A Review. Carbohydrate Polymers, 68, 587-597.

https://doi.org/10.1016/j.carbpol.2006.12.011 